Kalinkov: Yes, we have now some maps for the old Hubble counts. They are similar to the maps for Lick counts but not to Zwicky cluster maps.

Tammann: Do you find the same superclusters from two or more catalogues? If so are the observed identities above those expected from chance coincidences?

Kalinkov: Yes, there are at least 20 second-order cluster coincidences between Abe11 and Zwicky catalogues. The expected number of chance coincidences is 2 or 3 .

\title{
ON THE METHODS OF DISCOVERING GROUPS AND CLUSTERS OF GALAXIES
}

\section{B. I. Fessenko}

Groups. Even if real groups did not exist, the investigation of large regions of the sky will reveal other dense clumpings of galaxies. The usual estimate of the number of optical members in these clumpings will confirm their reality. But let us remember that a11 these groups are false. False groupings of galaxies should emerge in large numbers because of the effects of interstellar absorption and of variability of the observating conditions. Statistical considerations of the groups detected by Turner and Gott (1976, Ap. J., Suppl. Ser., 32, 409) leads to the conclusion that the percentage of false groups members is equal to $40 \%$ or more.

Clusters. Here the same remarks apply. At a given apparent limiting magnitude the distribution of galaxies (or real groups) as a function of distance from us has a single maximum. Near that maximum false clusters emerge with the greatest probability. With a larger limiting magnitude for galaxies in the sample, more distant false clusters should be obtained. Their angular diameters decrease in the same way as if the clusters were real ones. Statistical investigations of the Lick counts and other sources of data on the apparent distribution of galaxies lead me to the conclusion that a substantial fraction of the clusters of Zwicky and Abe11 are false clusters.

\section{DISCUSSION}

de Vaucouleurs: We do not understand how you could separate out the galactic extinction variance by restricting your study to $b \geqslant 60^{\circ}$ (i.e. $\operatorname{cosec} b \approx 1$ ).

Fessenko: In the analysis of the Lick counts, absorption of light was estimated not by means of a cosec law but by a special differential method of processing unreduced galaxy counts.

Bolton: Is the absorption you are considering colour dependent or nonselective? 
Fessenko: New data on the luminosities, linear dimensions and the B-V colour indices for ScI galaxies by Rubin, Ford and others enabled us to obtain values for the light absorption coefficient which exceeded 0.36 .

\title{
THE AUTOCORRELATION ANALYSIS OF DEEP GALAXY SAMPLES
}

\author{
S. Phillipps \\ (read by G. Efstathiou)
}

The two point correlation function $w(\theta)$ has been evaluated for the galaxies measured by the COSMOS machine at the Royal Observatory, Edinburgh, in an area of about 2 square degrees on a 2 hour exposure $J$ plate and a 2 hour exposure R plate (Phillipps, S., Fong, R., Ellis, R.S., Fal1, S.M. and MacGillivray, H.T., 1977, Mon. Not. R. astr. Soc., in press). In each case $w(\theta)$ is found to be in agreement with the form $\mathrm{w}=\mathrm{A} \theta^{-0.8}$ found previously by Peebles and coworkers. Since the samples are not magnitude limited the selection function, i.e. the distribution in distance, was determined by using models of the galaxy distribution to fit the observed angular diameter counts. However, when these selection functions are used to scale the amplitudes found for our samples, the amplitudes are found to be lower than those expected from Peebles' results by a factor of about 3. We consider that this is likely to be due to a lack of clusters in the small area of sky which we have studied: analysis of further areas should show whether this is the case.

\section{DISCUSSION}

Huchra: Have you tried to obtain an estimate of the limiting magnitude of your plates independent of your very complicated procedure?

Efstathiou: No.

Longair: What do you estimate the typical redshifts of the galaxies you are studying to be?

Efstathiou: Typically about 0.2 to 0.3 . 